\title{
Disulgación de la nanotecnología y papel de la sociedad en España, Estados Unidos y México
}

\author{
Miguel García Guerrero* \\ Guillermo Foladori**
}

\begin{abstract}
¿La divulgación científica explica de manera resumida y simple lo que es amplio y complejo? Esto es lo que comúnmente se entiende por disulgación científica. Sin embargo, según los procedimientos y el tipo de contenido, la divulgación científica predispone favorable o desfavorablemente al receptor. En lo que sigue of recemos el resultado preliminar de una metodología aplicada a algunos casos de divulgación científica sobre nanotecnologías en Estados Unidos, España y México.
\end{abstract}

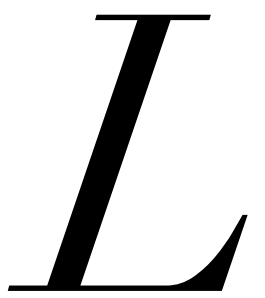

A CIENCIA y tecnología (Сут) representan una paradoja para la sociedad moderna: son elementos esenciales para el desarrollo

* Estudiante del Doctorado en Estudios del Desarrollo y miembro del Museo de Ciencias de la Universidad Autónoma de Zacatecas, México. Correo:miguel@grupoquark.com.

** Docente-investigador de la Unidad Académica en Estudios del Desarrollo de la Universidad Autónoma de Zacatecas, México. Coordinador de la Red Latinoamericana de Nanotecnología y Sociedad.
- por su capacidad creadora de innovaciones para dinamizar la economía o responder a problemas sociales - , pero se han vuelto tan especializadas, que la mayoría de las personas no tiene una idea clara de sus principales avances, la forma de aprovecharlas o cómo afectarán sus vidas. En palabras de Sagan: "Vivimos en una sociedad absolutamente dependiente de la ciencia y tecnología y aun así nos las hemos arreglado para que casi nadie entienda la ciencia y tecnología". ${ }^{.}$

A pesar de lo anterior - como señala Lewenstein,$-{ }^{2}$ la CyT sólo existen en un contexto social y no podemos entender su desarrollo sin comprender tanto las condiciones sociales que las producen como el 
efecto científico-tecnológico que impacta y da forma a la sociedad. Entonces es tarea de la divulgación de la сут comunicar al público no experto los elementos técnicos inherentes a los nuevos avances y también los aspectos sociales, históricos, económicos, políticos y culturales asociados a ellos.

Para Alcíbar, ${ }^{3}$ la divulgación de la cyт selecciona, redirige, adapta y recrea conocimientos producidos en un ámbito especializado para que - una vez transformados - cumplan una función social en un contexto distinto. La manera como los divulgadores científicos presentan a la cyт, que no es igual a como la presentan los científicos y tecnólogos, tiene gran importancia porque orienta sobre cómo entender el camino que aquéllas están tomando y sus efectos sobre la sociedad; además la versión de la cyT de los divulgadores científicos no sólo llega al "público en general", sino que es la que la gran mayoría de agentes que participan en las políticas de cyт conoce. ${ }^{4}$ No es lo mismo, por ejemplo, que la divulgación de la cyT diga que la automatización de la fábrica de automóviles es un logro porque permite producir ıo veces más autos por hora, que decir que la automatización de la fábrica de automóviles provocará un desempleo de tantos obreros. Aunque los dos resultados pueden ser ciertos, el acentuar uno u otro puede tener implicaciones muy diferentes. Los estudios de comunicación pública de la Сут nos ofrecen tres modelos principales para caracterizar la forma de relacionarse con el público: el déficit, el interactivo y el compromiso público.

El uso del término déficit se refiere a una transmisión de conocimiento desde los científicos al público ignorante que se limita a asimilar pasivamente el conocimiento. ${ }^{5}$ Con frecuencia adopta un enfoque propagandístico que muestra una perspectiva llena de bondades y carente de defectos (o riesgos). Este enfoque también puede corresponder al intento de la comunidad científica por conseguir recursos. ${ }^{6}$

En contraparte, se ha propuesto el modelo interactivo, o de diálogo, donde la divulgación se da a través de una interacción con el receptor basada en aspectos como información de riesgos, contexto social y el interés de los diferentes sectores del público. ${ }^{7}$ Los diferentes sectores del público asumen un papel activo y el proceso de divulgación termina siendo una creación conjunta entre el conocimiento científico y el contexto en el cual se transmite. ${ }^{8}$

El modelo democrático planteado por Durant ${ }^{9}$ sirve de base para lo que ahora ha llegado a conocerse como compromiso público (public engagement) e implica la creación de mecanismos que faciliten un debate público informado - con la participación de expertos técnicos, expertos no técnicos, representantes de grupos de interés y ciudadanos o "gente común" - como base para políticas públicas democráticas sustentables que cuenten con la confianza del público..$^{10}$ Se trata de la modalidad menos común, pues requiere la participación comprometida de instituciones capaces de moldear el avance de la cyт.

El modelo de divulgación adoptado cobra relevancia cuando se trata de sistemas científico-tecnológicos emergentes porque pueden llegar a moldear su desarrollo. Tal es el caso de las nanotecnologías, un sistema que combina elementos novedosos, complejos y disruptivos.

\section{Nanotecnologías}

Las NT representan un concepto paraguas, de ahí el uso plural de "nanotecnologías", que agrupa avances tecnológicos desarrollados desde diferentes campos: microscopía, electrónica, química, medicina, biotecnología y farmacología, por mencionar algunos. El único elemento en común para tal diversidad de disciplinas es el tamaño; todas trabajan con materiales que cuentan con al menos una dimensión en el rango de entre I y Ioo nanómetros. Un nanómetro equivale a Io $^{-9}$ metros, es decir, la millonésima parte de un milímetro. Mihail Roco, uno de los líderes del impulso a las NT en Estados Unidos, las define como:

La habilidad para controlar y reestructurar la materia al nivel atómico y molecular, en el rango aproximado de I-IOo nm, así como explotar propiedades y fenómenos distintos en esa escala [...]. La meta es crear materiales, dispositivos y sistemas con propiedades y funciones fundamentalmente nuevas a través de la ingeniería en su pequeña estructura. ${ }^{\prime \prime}$

La principal característica que hace especial a la escala "nano" es que las propiedades físicas y químicas de los materiales en este tamaño son dramáticamente diferentes a las de objetos más grandes del mismo material, lo cual permite la creación de artefactos y materiales con funciones novedosas. Las NT ya nos ofrecen nuevos dispositivos para la generación y almacenamiento de energía, materiales más ligeros que el acero pero mucho más resistentes, electrónicos cada vez más 
pequeños y potentes, así como medicamentos experimentales que pueden enviarse a objetivos específicos en el cuerpo. Y no olvidemos la base material del potencial revolucionario: los microscopios de proximidad - de fuerza atómica (AFM) y de barrido de túnel (STM) - que por primera vez le permitieron al ser humano visualizar y manipular la materia con precisión atómica.

De 2000 a 20II, el sector público mundial destinó 67.5 mil millones de dólares para apoyar investigaciones en nanotecnologías ${ }^{12} \mathrm{y}$ podemos hacernos una idea del avance comercial a través del inventario de productos de consumo final realizado por el "Proyecto sobre Nanotecnologías Emergentes"13 en el Woodrow Wilson International Centre for Scholars en Estados Unidos. Al II de mayo de 20r4, el inventario incluía I,88o productos.

Toda tecnología implica riesgos y los beneficios de las nanotecnologías deben considerarse frente a los peligros que implican. ${ }^{14}$ Entre 2000 y 20ro, la Nanotechnology Citizen Engagement Organization (Nanoceo) acumuló un banco de información con 176 artículos científicos sobre los riesgos de los nanotubos de carbono, igo sobre la nanoplata y 70 sobre las nanopartículas de dióxido de titanio. ${ }^{\text {.5 }}$ Día con día se realizan avances en la caracterización tóxica de nanopartículas, pero la industria en su prisa por llevar productos al mercado, rebasa la de la comunidad toxicológica para realizar pruebas de riesgo con nanopartículas. ${ }^{16}$

Aquí la divulgación resulta fundamental para realizar una reflexión de qué hacer frente a esta situación: ¿se mantiene el enfoque de introducir nuevas tecnologías al mercado y luego ver si son peligrosas o se busca una acción precautoria que sólo permita su ingreso al mercado cuando han probado ser seguras? ¿Se han estudiado otras alternativas para solucionar un problema, antes de pensar en introducir nuevas tecnologías? Estas son decisiones trascendentes para la sociedad desde el punto de vista de la salud, la innovación y la economía. Sin embargo, no siempre se involucra a los diferentes agentes en debates de esta naturaleza. Los gobiernos han usado los procesos de divulgación como una validación - tipo déficit - para sus estrategias en NT.

En gran medida la participación social se ha visto limitada por la falta de visibilidad de las NT, pues muchos agentes ni siquiera saben de su existencia: a diferencia de otros importantes sistemas tecnológicos que eran perfectamente identificables - como el generador eléctrico, la máquina de vapor o los aparatos electrónicos--, las NT son invisibles: son tan pequeñas que no podemos verlas. La mayoría de las personas no es- tán familiarizadas con el concepto de nanotecnologías. ${ }^{17}$ Un meta-análisis de 22 encuestas que se realizaron entre 2002 y 2009 en Estados Unidos, Canadá, Europa y Japón encontró bajos niveles de familiaridad con las NT, pero con una visión de beneficios que se imponen a los riesgos de 3 a I, aunque un 44 por ciento de las personas no tenían posición. ${ }^{18}$ Este alto grado de desconocimiento nos lleva a preguntar si se está haciendo divulgación para corregir esta situación y cuáles son las características de las estrategias que se llevan a cabo.

\section{Estudio de tres países: Estados Unidos, España y México}

El presente trabajo realiza un análisis preliminar de estrategias de divulgación en tres países a través de tres medios específicos de divulgación. Los países elegidos son Estados Unidos, como pionero y líder internacional en NT; España, líder iberoamericano en cuanto a estrategias de divulgación y educación en el tema nano; y México, como un país periférico que intenta subirse a la ola de las NT. Los medios fueron seleccionados considerando tres formas distintas de plantear la información y relacionarse con los públicos: libros, exposiciones interactivas y videos documentales. A continuación ofrecemos un contexto general de la divulgación de las NT en cada país, el cual nos sirve de preámbulo para abordar un estudio piloto de algunas estrategias en los tres países.

\section{Estados Unidos}

Estados Unidos es el referente mundial en cuanto al impulso de las NT; sus estrategias abrieron camino y marcaron una tendencia a nivel internacional desde la década de r99o. En 200o, este país estableció la National Nanotechnology Initiative (NNI), programa que desde entonces ha invertido más de I9 mil millones de dólares. Como parte del programa Broader Impacts de la National Science Foundation (NSF) - iniciado en 1997- todos los proyectos de investigación que reciben financiamiento de la NSF deben dedicar parte de sus recursos a aspectos sociales de la cyt, entre los que destacan las actividades de divulgación del tema investigado.

La NNI contempló un apartado de comunicación pública de las NT y el compromiso creció considerablemente en 2005, cuando la NSF otorgó el apoyo más grande para una estrategia de divulgación en su his- 
toria: 20 millones de dólares para crear la Red de Educación Científica No Formal a Escala Nano (Nanoscale Informal Science Education Network, NISE vet). ${ }^{19}$ El financiamiento fue renovado en 20 o para continuar el proyecto por otros cinco años, con lo que a la fecha suma más de 4I millones de dólares. La NISE Net ha creado una serie de materiales - exposiciones, talleres, videos y libros - que comparte de forma libre a través de su sitio web y del sitio www.whatisnano.org; además se han impulsado foros de compromiso público. A la fecha, cientos de museos de ciencia y otras organizaciones de educación científica no formal han participado en las diferentes actividades de la NISE net..o

Paralelamente, la National Nanotechnology Infrastructure Network (NNIN), organización integrada por centros avanzados de NT en I 4 grandes universidades, ha promovido actividades de divulgación como la revista Nanooze y la exposición del mismo nombre, así como la creación de un manual de talleres demostrativos, el museo móvil "Nanoexpress" y talleres locales en sus diferentes sedes. Además de estos esfuerzos de coordinación nacional que han sido aprovechados por cientos de instancias locales, existen numerosas estrategias independientes de divulgación de NT en Estados Unidos: desde esfuerzos de centros académicos hasta actividades individuales.

España

España se ubicaba como el séptimo país en términos de producción científica en NT a nivel mundial, y el más productivo en el contexto iberoamericano. ${ }^{21} \mathrm{La}$ capacidad española nace con el acceso privilegiado de grupos de investigación al trabajo de microscopía de proximidad (STM y AFM) en la década de $1980,{ }^{22}$ y se ha visto fortalecida por la organización de los investigadores en la Red NanoSpain, la cual cuenta con más de 3io grupos inscritos y sirvió de catalizador para darle trascendencia a las NT dentro de los planes nacionales de $\mathrm{I}+\mathrm{D}+\mathrm{i}$ entre 2004 y $201 \mathrm{I}{ }^{23}$

Sin embargo, esto no se ha reflejado en un trabajo sistemático respecto a la divulgación. La Fundación Española para la Ciencia y Tecnología (FECYT), institución pública creada en 200I y que tiene a su cargo las estrategias de divulgación de la cyт, no ha realizado hasta el momento ningún esfuerzo para articular el trabajo de divulgadores e investigadores a escala nacional. La única estrategia de la FECYT para la divulgación de las NT ha sido la elaboración de la Unidad Di- dáctica de Nanociencia y Nanotecnología (2008). A nivel personal, y con el respaldo del Consejo Superior de Investigación Científica (CSIC), destaca la labor de Pedro Serena Domingo, del Instituto de Ciencia de Materiales en Madrid, quien ha participado en la publicación de tres libros de divulgación de las NT, ha promovido la creación de la exposición "Un paseo por el nanomundo", ha desarrollado el taller "Explorando el nanomundo" y fue el líder de la serie de documentales ¿Qué sabemos de nanotecnología?, realizados de forma conjunta por el CSIC y la Universidad Nacional de Educación a distancia.

\section{México: un rumbo incierto}

México ha comprado la receta de los organismos internacionales de apostar a las NT como agentes de competitividad; sin embargo, esto se realiza sin una estrategia y con recursos paupérrimos para los estándares internacionales. La política mexicana en materia de NT está plagada de buenas intenciones, pero no existe una verdadera estrategia rectora para el trabajo en el tema a nivel nacional. ${ }^{24}$ Entre las pocas acciones que se han promovido, en 2009 el Consejo Nacional de Ciencia y Tecnología (Conacyt) contribuyó a la creación de una red nacional para conectar a los más de 5o centros de investigación en NT del país. ${ }^{25}$

Hasta el momento, el Conacyt no cuenta con una política ni una estrategia nacional de divulgación de la Сут en general, mucho menos para el caso de las NT. Las acciones de comunicación pública de las NT parten de iniciativas aisladas por parte de investigadores y centros académicos. Destaca en este sentido el trabajo de Ciencia Pumita, liderada por Noboru Takeuchi desde el Centro de Nanociencias y Nanotecnologías de la Universidad Nacional Autónoma de México (UNAM) en Ensenada, que ha publicado una serie de libros y documentales.

Cuadro i. Estrategias de divulgación CIENTÍFICA EN PAÍSES SELECCIONADOS

\begin{tabular}{ccccc}
\hline País & Libros & $\begin{array}{c}\text { Exposiciones } \\
\text { Talleres }\end{array}$ & $\begin{array}{c}\text { Docu- } \\
\text { mentales }\end{array}$ & Total \\
\hline $\begin{array}{c}\text { Estados } \\
\text { Unidos }\end{array}$ & 25 & 18 & 9 & 52 \\
\hline España & 10 & 4 & 2 & 16 \\
\hline México & 5 & 3 & 6 & 14 \\
\hline & 40 & 25 & 17 & 82 \\
\hline
\end{tabular}




\begin{tabular}{|c|c|c|c|c|}
\hline Nombre & Autor/Lider & Institución & Medio & País \\
\hline Radical abundance & Eric Drexler & University of Oxford & Libro & EE.UU \\
\hline Alice in nanoland & $\begin{array}{l}\text { Leigha Horton } \\
\text { Stephanie Long }\end{array}$ & $\begin{array}{l}\text { NISE Net/ Science } \\
\text { Museum of Minnesota }\end{array}$ & Libro & EE.UU \\
\hline Nanociencia y nanotecnología & $\begin{array}{l}\text { José Martín } \\
\text { Gago }\end{array}$ & FECYT & Libro & España \\
\hline La nanotecnología & Pedro Serena & CSIC & Libro & España \\
\hline Nanociencia y nanotecnología & Noboru Takeuchi & UNAM & Libro & México \\
\hline El pequeño e incríble Nanomundo & $\begin{array}{l}\text { Noboru Takeuchi } \\
\text { Marisol Romo }\end{array}$ & UNAM & Libro & México \\
\hline Nano & $\begin{array}{l}\text { Rae Ostman } \\
\text { Ali Jackson }\end{array}$ & NISE vet/Sciencenter & $\begin{array}{l}\text { Expo/ } \\
\text { Taller }\end{array}$ & EE.UU \\
\hline $\begin{array}{l}\text { Nanotechnology Outreach } \\
\text { Demonstrations }\end{array}$ & $\begin{array}{l}\text { Nancy Healy } \\
\text { Joyce Palmer }\end{array}$ & NNIN & $\begin{array}{l}\text { Expo/ } \\
\text { Taller }\end{array}$ & EE.UU. \\
\hline Explorando el Nanomundo & Pedro Serena & $\operatorname{CSIC}$ & $\begin{array}{l}\text { Expo/ } \\
\text { Taller }\end{array}$ & España \\
\hline Dimensión Nano & Boaz Kogon & $\mathrm{ICN} 2$ & $\begin{array}{l}\text { Expo/ } \\
\text { Taller }\end{array}$ & España \\
\hline $\begin{array}{l}\text { Nanotechnology: what's the big } \\
\text { deal? }\end{array}$ & & $\begin{array}{l}\text { NISE Net/Oregon } \\
\text { Museum of Science and } \\
\text { Industry }\end{array}$ & Video & EE.UU. \\
\hline ¿Qué sabemos de nanotecnología? & Pedro Serena & CSIC/UNED & Video & España \\
\hline ¿Qué es la nanotecnología? & & UNAM & Video & México \\
\hline
\end{tabular}

El estudio PILOTO

En total, para los tres países, se detectó un universo de 82 estrategias de divulgación científica en los medios seleccionados: 4o libros, 25 exposiciones/talleres y 17 documentales que se distribuyen documentales (véase cuadro I).

De este total se seleccionaron aquellas que cuentan con apoyo de una organización oficial, que surgieron de una red de colaboración o se insertan en un proyecto más amplio de participación social en NT. El resultado fue: II de España (seis libros, tres exposiciones y dos documentales), 28 de Estados Unidos (I3 libros, io exposiciones y cinco documentales) y io de México (cuatro libros, dos exposiciones y cuatro documentales). Para efectos de un primer análisis piloto para poner a prueba la herramienta metodológica se tomaron algunos casos de cada país, que son los que exponemos en el cuadro 2.
Tras analizar las características de cada una de las estrategias, la información recabada se capturó en una matriz elaborada específicamente para caracterizar las estrategias de divulgación a través de los elementos de contexto, forma y contenido. De esta manera, es posible ubicar ciertas tendencias.

\section{Resultados}

En primer lugar, se analizó el contexto institucional que dio origen a la estrategia: el tipo de institución que asumió el liderazgo, las instituciones colaboradoras, aquellas que otorgaron el financiamiento y las que distribuyeron los productos. La gráfica i muestra que, por lo general, las estrategias provienen de instituciones públicas educativas como universidades, museos y centros de investigación, mientras que el financiamiento proviene en gran medida del sector gubernamental. En términos 


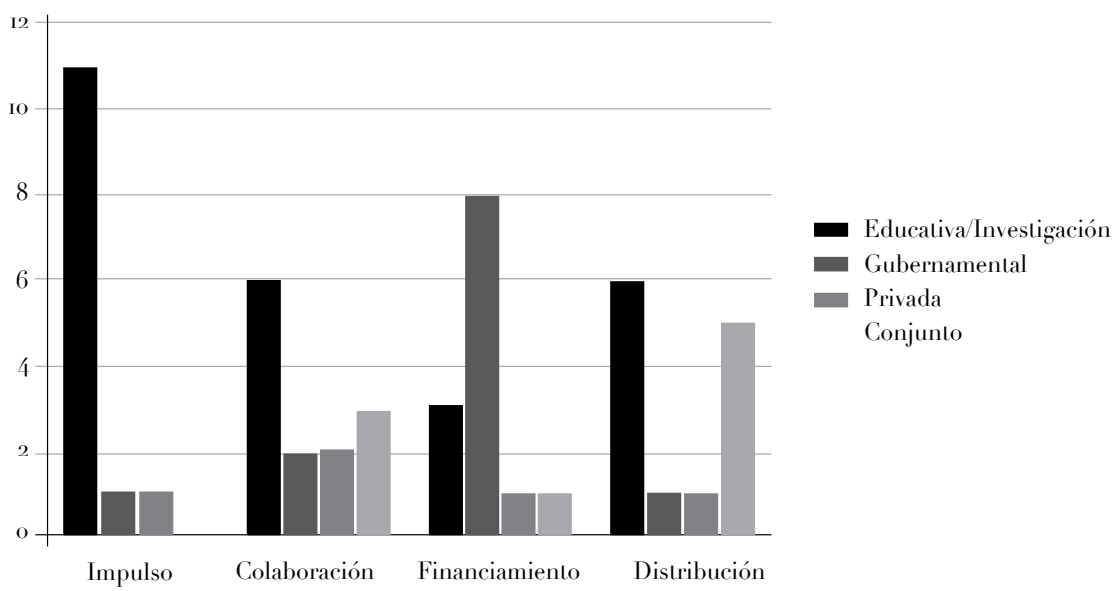

Fuente: Elaboración propia

\section{GrÁFICA 2}

Modelo de divulgación usado por las estrategias por país

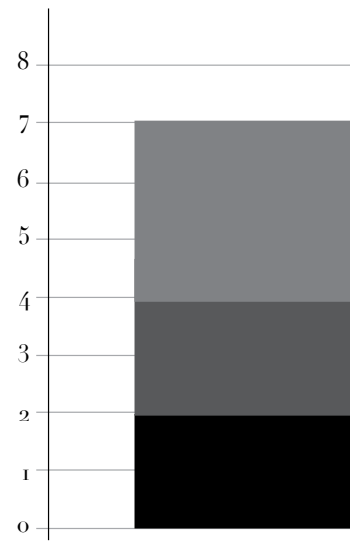

Déficit

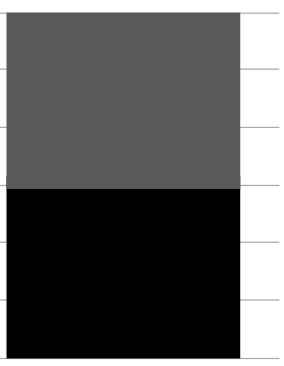

Diálogo
- México

España

- Estados Unidos

Fuente: Elaboración propia.

Gráfica 3.

Contenido abordado en estrategias por país

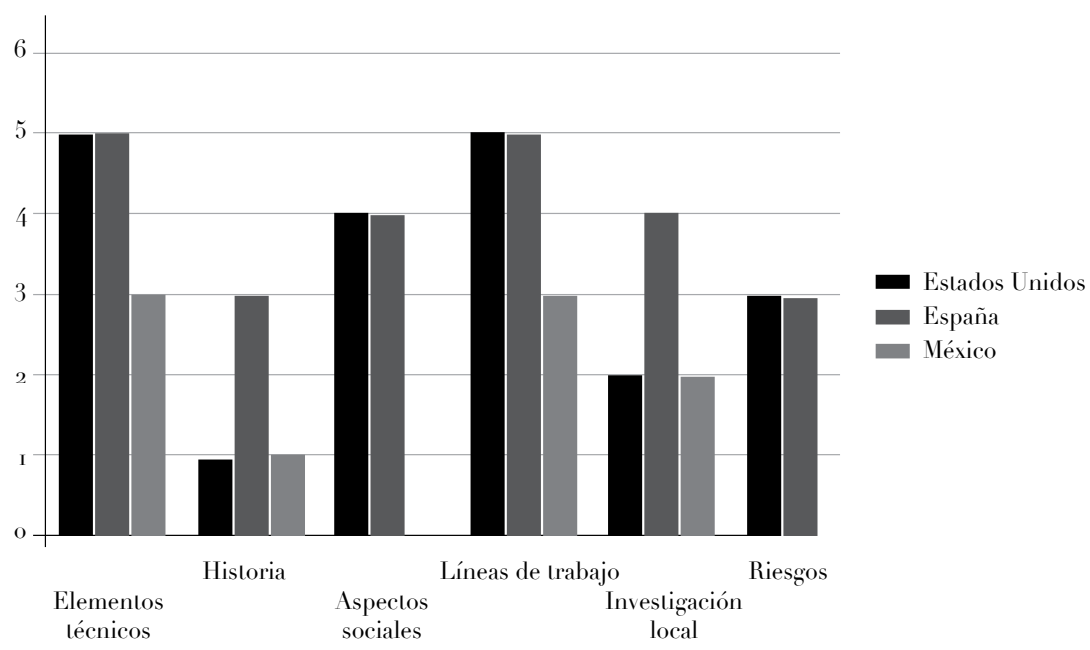

Fuente: Elaboración propia 
generales, la participación del sector privado es muy baja, se limita esencialmente a las editoriales involucradas en la publicación y distribución de los libros.

En cuanto al modelo de divulgación utilizado en las estrategias (véase gráfica 2), encontramos una pequeña ventaja en las estrategias que trabajan tipo déficit $(7)$ sobre aquellas que buscan establecer un diálogo (6). Esto puede explicarse si consideramos que sólo tres de los 13 líderes de los proyectos se dedican a la divulgación como su actividad principal, mientras que de los demás, nueve son investigadores y uno administrador de un centro científico.

La gráfica 3 nos permite hacer un contraste del contenido de las diferentes estrategias: encontramos que la totalidad de las estrategias incorpora los elementos técnicos generales que caracterizan a las NT y la mayoría hablan de las principales líneas de trabajo en las que se avanza; sin embargo la presencia de historia, aspectos sociales y riesgos es mucho más baja - especialmente en México, donde el abordaje del tema es nulo-. En esencia, las estrategias que incorporan estos elementos son aquellas que han alcanzado un mayor grado de madurez y que involucran a especialistas tanto en el tema de NT como en ciencias socia- les y a divulgadores profesionales, para trascender una visión técnica/paradigmática y ofrecer una narrativa integral capaz de incorporar lo científico-tecnológico a la par de lo social.

\section{Conclusiones}

Es imposible separar a la cyT de su contexto social y de la forma en que se moldean mutuamente, sin embargo muchas estrategias de divulgación de NT dejan fuera estos aspectos desestimando el aporte que los diferentes sectores sociales pueden hacer al proceso de comunicación y el valor que tienen para moldear el rumbo de las NT. Los casos analizados muestran, implícitamente, que la divulgación es cuestión de científicos y tecnólogos, sin tomar en cuenta las necesidades y preocupaciones de la sociedad.

Sin embargo, ya existen experiencias en que la interacción de científicos con diferentes agentes y la colaboración entre especialistas de NT con divulgadores profesionales permite trascender aquella visión limitada. De esta forma, se empieza a abrir la puerta para una creciente participación social en la definición de una ruta nanotecnológica.

\section{- notas $\cdot-$}

' Carl Sagan (2006), Conversations with Carl Sagan, University Press of Mississippi.

"Bruce Lewenstein (2005), "What Counts as a 'Social and Ethical Issue' in Nanotechnology?”, Hyle, II(I), p. 6.

${ }^{3}$ Miguel Alcíbar Cuello (2004), "La divulgación mediática de la ciencia y la tecnología como recontextualización discursiva", Anàlisi: Quaderns de comunicació i cultura, 3г, pp. 45 .

4 Aant Elzinga y Andrew Jamison (1995), "Changing Policy Agendas in Science and Technology", en Handbook of Science and Technology Studies, Londres, Sage, p. 574.

${ }^{5}$ John Durant (1999), "Participatory Technology Assessment and the Democratic Model of the Public Understanding of Science", Science and Public Policy, 26(5), p. 3ı́.

${ }^{6}$ Bruce Lewenstein (1995), "Science and the Media", en Handbook of Science and Technology Studies, Londres, Sage, pp. 345.

IIbidem, p. 348 .

${ }^{8}$ Alan Gross (I994), "The roles of Rhetoric in the Public Understanding of Science", Public Understanding of Science, 3(I), pp. 5-6.

${ }_{9}$ Durant, op. cit.

'о Ibidem, p. 3І5.

"Mihail Roco (20II), "The Long View of Nanotechnology Development: The National Nanotechnology Initiative at io Years", Journal of Nanoparticle Research, 13(2), p. 428.

${ }^{12}$ Científica (2011), "Global Funding of Nanotechnologies 20II Edition". http://www.cientifica.com/research/market-reports/nanotech-funding-20II

${ }^{13} \mathrm{http}: /$ www.nanotechproject.org/cpi/products/
${ }^{14}$ Adam Corner y Nick Pidgeon (2012), "Nanotechnologies and Upstream Public Engagement. Dilemmas, Debates and Prospects?", en The Social Life of Nanotechnology, Nueva York, Routledge Studies in Science, Technology and Society, p. I69.

${ }^{15}$ Foladori e Invernizzi (2012), op. cit., p. 17.

${ }^{16}$ David Berube, Brenton Faber, Dietram Scheufele, Christopher Cummings, Grant Gardner, Kelly Martin, Michael Martin y Nicholas Temple (2010), Communicating Risk in the 21st Century: The Case of Nanotechnology, p. 19.

17 Phil Macnaghten (2010), "Researching Technoscientific Concerns in the Making: Narrative Structures, Public Responses, and Emerging Nanotechnologies", Environment and Planning $A$, 42(I), p. 24.

${ }^{18}$ Mihail Roco, Barbara Harthorn, David Guston y Philip Shapira (2OI), "Innovative and Responsible Governance of Nanotechnology for Societal Development, Journal of Nanoparticle Research, i3 (9), p. 3563.

${ }^{19}$ David Chittenden (2011), "Roles, Opportunities and Challenges-Science Museums Engaging the Public in Emerging Science and Technology, Journal of Nanoparticle Research, i3, p. I554.

${ }^{20}$ Ibidem, p. I554.

${ }^{2}$ OEI (2009), La nanotecnología en Iberoamérica, situación actual y tendencias, Organización de Estados Iberoamericanos.

${ }^{22}$ Pedro Serena y Joaquín Tutor (2011), "La divulgación y la formación de la nanociencia y la nanotecnología en España: un largo camino por delante", Mundo Nano, 4(2), pp. 48-49. 
${ }_{23}^{23}$ Pedro Serena (2014), "Divulgación de nanotecnologías en España”, entrevista personal.

${ }^{2}$ Edgar Záyago y Guillermo Foladori (2010), "La nanotecnología en México: un desarrollo incierto", Economía, Sociedad y Territorio, X (32), p. ı丂̆.
${ }^{25}$ Guillermo Foladori e Noela Invernizzi (2013), "Inequality Gaps in Nanotechnology Development in Latin America”, Journal of Arts and Humanities, 2(3), p. 36.

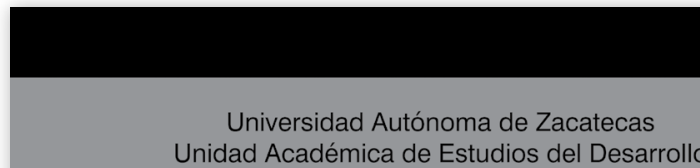

II Seminario de Universitarios por el Desarrollo La Otra Toma de Zacatecas: Un Siglo Después

Conferencia magistral

"Dominio agroalimentario y

la lucha por el poder"

Dra. Blanca Rubio

Instituto de Investigaciones

Sociales

Universidad Nacional Autónoma de México

Presentación del libro

El dominio del hambre

Crisis de hegemonía $y$ alimentos

Moderador:

Dr. Rodolfo García Zamora

Unidad Académica de Estudios del Desarrollo

\section{ENTRADA LIBRE}

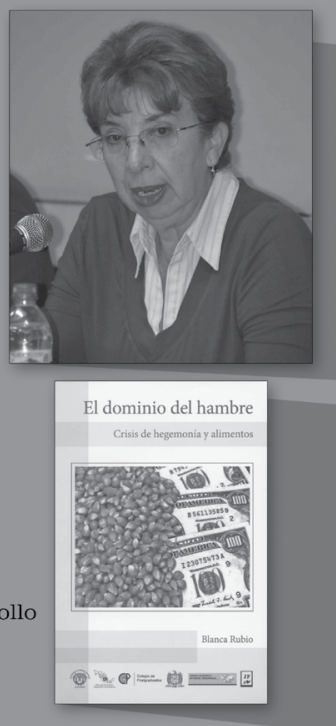

\section{martes 18 de noviembre de 2014}

\section{0:00 a.m.}

Auditorio de Estudios

del Desarrollo

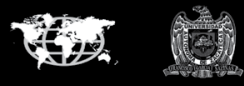

Universidad Autónoma de Zacatecas Unidad Académica de Estudios del Desarrollo

II Seminario de Universitarios por el Desarrollo La Otra Toma de Zacatecas: Un Siglo Después

Conferencia magistral

Ciclos y oscilaciones del pensamiento latinoamericano desde el "Manifiesto"

a su más reciente conversión

Carlos Mallorquin

Unidad Académica de Estudios del

Desarrollo, UAZ

\section{ENTRADA LIBRE}

miércoles 19 de noviembre de 2014 10:00 a.m.

Auditorio de Estudios del Desarrollo 